\title{
Alterstice
}

Revue internationale de la recherche interculturelle

International Journal of Intercultural Research

Revista International de la Investigacion Intercultural

\section{Parents entendants d'enfants sourds en France : récits de mères illustrant les écarts entre discours officiels et pratiques des professionnels face à la diversité des modèles de communication existants}

\section{Pauline Rannou}

Volume 7, numéro 2, 2017

Diversité(s) au coeur des politiques et des pratiques : entre l'Europe et l'Afrique

URI : https://id.erudit.org/iderudit/1052570ar

DOI : https://doi.org/10.7202/1052570ar

Aller au sommaire du numéro

Éditeur(s)

Alterstice

ISSN

1923-919X (numérique)

Découvrir la revue

Citer cet article

Rannou, P. (2017). Parents entendants d'enfants sourds en France : récits de mères illustrant les écarts entre discours officiels et pratiques des professionnels face à la diversité des modèles de communication existants. Alterstice, 7(2), 67-76. https://doi.org/10.7202/1052570ar

\section{Résumé de l'article}

Cet article, issu de notre recherche doctorale, propose d'analyser, dans le cadre d'entretiens semi-directifs réalisés en France, les témoignages de parents entendants découvrant la surdité de leur enfant. Nous avons analysé le parcours de ces parents, du dépistage néonatal à l'annonce de la surdité, leurs attentes en termes d'informations de la part des professionnels puis en termes d'accompagnement. Si les textes officiels prônent la prise en compte de la diversité des situations familiales et si plusieurs options linguistiques et éducatives ou cliniques sont offertes aux parents, traduisant divers modèles de communication à leur disposition, la réalité des pratiques hiérarchisées et discontinues semble loin de ces discours officiels de choix, de conseil et de suivi personnalisé. 


\section{7}

ARTICLE THÉMATIQUE

\section{Parents entendants d'enfants sourds en France : récits de mères illustrant les écarts entre discours officiels et pratiques des professionnels face à la diversité des modèles de communication existants}

Pauline Rannou ${ }^{1}$

\section{Résumé}

Cet article, issu de notre recherche doctorale, propose d'analyser, dans le cadre d'entretiens semi-directifs réalisés en France, les témoignages de parents entendants découvrant la surdité de leur enfant. Nous avons analysé le parcours de ces parents, du dépistage néonatal à l'annonce de la surdité, leurs attentes en termes d'informations de la part des professionnels puis en termes d'accompagnement. Si les textes officiels prônent la prise en compte de la diversité des situations familiales et si plusieurs options linguistiques et éducatives ou cliniques sont offertes aux parents, traduisant divers modèles de communication à leur disposition, la réalité des pratiques hiérarchisées et discontinues semble loin de ces discours officiels de choix, de conseil et de suivi personnalisé.

\section{Rattachement de l'auteure \\ ${ }^{1}$ Laboratoire PREFics EA 7469 (Pôle de recherche Francophonies, Interculturel, Communication, Sociolinguistique), Université de Rennes 2, France}

\section{Correspondance}

pauline.rannou@gmail.com

\section{Mots clés}

surdité, parcours des parents, diversité des modèles de communication, discours officiels, pratiques des professionnels, écarts

\section{Pour citer cet article}

Rannou, P (2017). Parents entendants d'enfants sourds en France : récits de mères illustrant les écarts entre discours officiels et pratiques des professionnels face à la diversité des modèles de communication existants. Alterstice, $7(2), 67-76$. 


\section{Introduction}

Le dépistage néonatal de surdité, généralisé dans les maternités depuis $2012^{1}$, est venu modifier la découverte, par les parents, de la surdité de leur enfant. L'annonce du diagnostic par les médecins est suivie de nombreuses interrogations, concernant notamment les possibilités de construire de nouvelles modalités de communication entre les parents et leur enfant. Cette contribution s'inscrit dans le cadre d'une thèse ${ }^{2}$ ayant pour objet le parcours de parents entendants, depuis l'annonce de la surdité de leur enfant jusqu'aux choix de communication (oral-vocal, signé, multimodal, bilingue) et de scolarisation. L'idée d'interroger uniquement des parents entendants (et non sourds) est liée au fait que 90 à $95 \%$ des enfants sourds naissent de parents entendants (Jones, 1989), qui constituent de ce fait la très grande majorité des parents d'enfants sourds. Ces entretiens ont eu lieu en intégralité en France et donnent à voir des expériences contrastées, qui nous ont amenée à remettre en question, dans notre recherche, les notions de handicap, d'identité, de langue et de culture sourde. En parallèle, un axe sociodidactique croise les discours d'enseignants spécialisés, de directeurs et d'acteurs éducatifs d'établissements dits " ordinaires ", " spécialisés » et " bilingues », en France et aux États-Unis, afin de saisir la diversité des approches pédagogiques dans ces deux pays et de proposer des pistes d'accompagnement destinées aux enseignants français accueillant des élèves sourds dans leur classe.

Nous nous intéresserons particulièrement ici aux discours des familles, à travers les récits de parents entendants. Cet article est articulé en quatre parties. La première partie expose les ancrages théoriques et méthodologiques de notre recherche et introduit le rôle central des parents dans le(s) choix entre différents modèles de communication pour et avec leur enfant. Nous développons par la suite, en deux étapes distinctes mais complémentaires, le parcours de parents entendants interrogés confrontés au diagnostic: d'abord l'annonce de la surdité puis l'information donnée par les équipes médicales, à travers les divers modèles de communication proposés et les choix que les parents doivent faire. La conclusion de cet article, loin de venir répondre aux questionnements nombreux engendrés par ces situations perturbantes, voire traumatisantes, pour les parents, tente toutefois d'apporter des pistes possibles pour mieux informer et mieux accompagner les parents.

\section{Les parents entendants au cœur d'un débat}

Pour les parents entendants, l'annonce de la surdité d'un enfant ouvre des interrogations totalement inédites concernant la communication entre leur enfant et eux, et plus largement entre leur enfant sourd et " les autres " (Dagron, 1999). Les enfants sourds de parents entendants se révèlent, dans ce cadre, en tant que figures d'une altérité à deux niveaux : ils sont hors de la " communauté » entendante majoritaire, dont la dénomination n'existe qu'à travers les discours relatifs à la surdité, et par là même hors de leur communauté familiale entendante. En effet, « en général, les parents entendants ignorent tout du monde de la surdité et lorsqu'ils découvrent que leur enfant est sourd, ils ne voient plus ce dernier communiquant selon ses propres modalités à la façon dont ils l'ont toujours vu, mais ils le voient privé de parole et de moyens de communication, la surdité étant essentiellement pour eux synonyme de mutité » (Bouvet, 2004, p. 75).

Les questionnements posés par la surdité aux parents entendants dévoilent la nécessité du choix de la langue, qui oriente de manière cruciale les modalités de communication de leur enfant et, plus tard, son parcours de scolarisation. Ce choix, rendu polémique par le clivage des positionnements déficitaires et anthropologiques de la surdité (Virole, 2006), place les parents au cœur d'un débat contradictoire entre souhait d'une communication par une même langue que la leur et possibilité d’une langue accessible immédiatement pour leur enfant; entre

\footnotetext{
${ }^{1}$ Ce dépistage, rendu systématique par l'arrêté du 23 avril 2012, comprend un examen de repérage des troubles de l'audition, proposé systématiquement, avant la sortie de l'enfant de l'établissement de santé dans lequel a eu lieu l'accouchement ou dans lequel l'enfant a été transféré (Légifrance, 2012).

${ }^{2}$ IReSP-15-Hand6-14. Cette recherche a bénéficié de l'aide de la Mission recherche de la Direction de la recherche, des études, de l'évaluation et des statistiques (MiRe-DRESS) et de la Caisse Nationale de Solidarité pour l'Autonomie (CNSA), dans le cadre de l'appel à projets lancé par l'IReSP en 2015. Thèse déposée en mai 2018.
} 
implantation cochléaire associée à une rééducation vocale ${ }^{3}$ en français et communication parfois dite « naturelle » (Bouvet, 2003) en langue des signes, langue que les parents entendants ne connaissent que très rarement.

\section{Méthodologie de recherche : choix d'une approche plurielle}

Nous présentons, pour cet article, les témoignages d'un petit nombre de parents entendants, que nous avons interrogés en France et qui « racontent » le parcours du protocole de dépistage de surdité en maternité jusqu'à l'annonce de la surdité de leur enfant. Chacun des entretiens a été conduit selon une trame commune comportant des thèmes généraux (annonce, information, appareillage, communication, scolarisation), qui nous ont permis d'identifier la diversité des situations, des solutions et des choix faits par les familles (tableau 1).

Tableau 1 : Trame d'entretiens auprès des parents entendants

\begin{tabular}{|c|c|}
\hline Thèmes & Relances \\
\hline Présentation & $\begin{array}{l}\text { Présentation de la personne enquêtée / Présentation } \\
\text { de la famille / nombre d'enfants / âge des enfants... }\end{array}$ \\
\hline \multirow{2}{*}{ Parcours familial } & Lien avec la surdité auparavant? \\
\hline & Si oui, quelle communication? \\
\hline \multirow{3}{*}{ Dépistage de surdité } & Combien de temps après la naissance \\
\hline & Conditions de dépistage / combien de tests PEA? \\
\hline & $\begin{array}{l}\text { Si pas dépistage : Comment avez-vous appris la surdité de } \\
\text { votre enfant? }\end{array}$ \\
\hline \multirow{2}{*}{ Annonce de la surdité } & Qui annonce (médecin, infirmière, interne...)? \\
\hline & Dans quelles conditions? Votre ressenti sur l'annonce. \\
\hline \multirow{3}{*}{$\begin{array}{l}\text { Accompagnement suite à } \\
\text { l'annonce }\end{array}$} & Que se passe-t-il après l'annonce? \\
\hline & Vous êtes-vous sentis accompagnés? \\
\hline & Accompagnement à la sortie de la clinique / hôpital. \\
\hline \multirow{3}{*}{ Aspect auditif } & Degré de surdité \\
\hline & Appareillé / Implanté / aucun? \\
\hline & Si oui, quel âge? \\
\hline \multirow{3}{*}{$\begin{array}{l}\text { Accompagnement et } \\
\text { Information }\end{array}$} & Informations sur les modes de communication? \\
\hline & Informations sur les modes de scolarisation? \\
\hline & Si accompagnement, qui informe? \\
\hline \multirow{5}{*}{ Communication } & Quelle communication? \\
\hline & Pour quelles raisons? \\
\hline & $\begin{array}{l}\text { Si pertinent : Vous êtes-vous déjà intéressés à la langue } \\
\text { des signes? Si oui pourquoi / si non pourquoi? }\end{array}$ \\
\hline & Connaissez-vous d'autres personnes sourdes? \\
\hline & Si oui : quelle communication? \\
\hline \multirow{3}{*}{ Scolarisation } & Quel système de scolarisation? Inclusion, bilingue... \\
\hline & Emploi du temps / orthophonie, psychologue?... \\
\hline & Combien d'heures d'orthophonie / motricité...? \\
\hline
\end{tabular}

Douze entretiens ont été menés auprès de familles ${ }^{4}$, plus précisément auprès des mères pour dix des douze entretiens effectués. Seuls eux entretiens ont été effectués auprès des deux parents. Le fait que la majorité des

\footnotetext{
${ }^{3}$ Les travaux de Millet (2004) ont amené à l'opposition « langue vocale/ langue gestuelle ». L' « oral », qui peut se référer à la langue des signes orale, est quant à lui opposé à l' " écrit ».
} 
entretiens aient été menés auprès des mères des enfants (et non auprès des pères ou en présence des deux parents) s'explique par le fait que le contact a été établi avec un premier " filtrage » : les spécialistes accompagnant les parents que nous avons interrogés ont échangé avec nous par courriel, les directeurs ne souhaitant pas divulguer d'information personnelle sans l'accord préalable des parents. C'est donc l'équipe médico-sociale qui a relayé la prise de contact avec les parents ayant accepté de me rencontrer et, dans la totalité des cas, seules les coordonnées de la mère de l'enfant m'ont été adressées ${ }^{5}$. Il est important de signaler qu'il s'agit ici d'enfants nés entre 2001 et 2012 qui présentent tous un degré de perte auditive que le corps médical qualifie de « sévère à profonde » et qui sont tous appareillés ou implantés.

La trame d'entretien - et les échanges spontanés avant et après l'entretien - consacre une part importante à l'aspect "médical » de la surdité, à travers le dépistage de la surdité, son annonce par les médecins et l'accompagnement qui a suivi l'annonce. Sont évoquées par la suite la diversité des modèles de communication puis celle des modèles de scolarisation possibles, les représentations associées à la surdité et les (éventuelles) difficultés rencontrées par les mères ${ }^{6}$.

L'analyse des entretiens de cette recherche a été effectuée à partir d'un choix d'outils jugés les plus appropriés à nos objectifs, et repose en ce sens sur une analyse "éclectique », pour reprendre les termes de Kerbrat-Orecchioni (2005), et non sur l'appartenance à un courant déterminé. Dans la logique de cette démarche " en éventail ", ou approche que nous pourrions également dénommer "plurielle ", nos analyses prennent dans un premier temps appui sur le logiciel Iramuteq, développé par Ratinaud ${ }^{7}$. Ce logiciel permet de faire des analyses statistiques sur des corpus de texte et reproduit la méthode de classification Alceste, décrite par Reinert (1990). La méthode Alceste vise au regroupement statistique des cooccurrences d'un texte et permet de développer les " mondes lexicaux" issus du corpus, autrement dit, schématise les liens que les termes lexicaux entretiennent au sein d'un corpus de plusieurs entretiens. Cette première phase d'analyse ${ }^{8}$ est ensuite associée à une phase d'analyse de contenu, que nous avons menée en nous inspirant des travaux de Laurence Bardin (2013) et Roger Mucchielli (2006) notamment.

\section{Analyses : écarts entre discours et institutions, réalités des parents et pratiques professionnelles}

\section{Du dépistage au temps de l'annonce : un parcours en lignes brisées}

Pour les parents entendants, le dépistage constitue la première entrée dans la surdité, une entrée biomédicale qui introduit la surdité comme une maladie néonatale de santé publique ${ }^{9}$, nécessitant un dépistage à deux jours (Meynard, 2016). Plusieurs familles interrogées dans le cadre de cette recherche font état du processus de dépistage, décrit par la plupart comme long et difficile.

\footnotetext{
${ }^{4}$ Au total, 61 entretiens semi-directifs ont été menés dans le cadre de cette recherche, auprès de parents entendants en France ainsi qu'auprès d'enseignants et directeurs d'établissements scolaires accueillant des élèves sourds en France et aux États-Unis.

${ }^{5}$ Nous avons ensuite fixé un rendez-vous, souvent en soirée après leur journée de travail, ce qui implique une fatigue supplémentaire au moment des échanges. Le lieu de l'entretien n'étant pas imposé, nous avons proposé une rencontre à l'université, au domicile des parents ou dans un lieu de leur choix pour chacun des entretiens. Dans chaque cas, la réponse a été celle du domicile des parents, par commodité. La durée de l'entretien a également conditionné le lieu du rendez-vous, puisque nous leur avions demandé une disponibilité d'environ deux heures afin d'aborder l'ensemble des thématiques que nous avions prévues. Les entretiens ont duré d'une à deux heures.

${ }^{6}$ L'ordre de la trame d'entretien n'a pas été nécessairement gardé au moment des entretiens et permet simplement le rappel (pour nous) des thématiques à aborder.

${ }^{7}$ http://www.iramuteq.org/documentation/html

${ }^{8}$ Nous ne présenterons pas ici les résultats des analyses lexicométriques menées avec le logiciel Iramuteq. Nous nous réfèrerons donc uniquement à nos analyses d'entretiens.

${ }^{9}$ Un certain nombre de rapports font état de l'appellation surdité permanente néonatale ou SPN en tant que maladie (Meynard, 2016 : 28). Voir le chapitre 4 de l'ouvrage, entièrement dédié à la "SPN ou la naissance d'une nouvelle maladie".
} 
Nous focalisons l'expérience du dépistage à l'annonce de surdité à travers le témoignage de Marie ${ }^{10}$, dont la fille, Barbara, est née en 2009 :

Marie : « en $2009 \downarrow$ j'ai accouché et donc ça fait partie du protocole de l'établissement / de faire les tests [tests d'autoémission] $\downarrow$ et puis là / elle [l'infirmière] a eu un avis réservé en nous disant que // les tests n'étaient pas concluants / voilà ce qu'elle nous a dit $\downarrow$ sans en dire plus mais en nous disant « il faudrait revenir dans quinze jours pour refaire les tests $\downarrow[\ldots]$ on s'est dit « petit bémol » $\downarrow$ on était super contents « oh bah tiens un petit test qui est pas concluant / ça ne fait rien on reviendra dans quinze jours $\downarrow$ on ne s'est pas alarmé plus que ça $\downarrow$

Enquêtrice : le mot surdité n'a pas été évoqué $\downarrow$

Marie : du tout $\downarrow$ non non / du tout $\downarrow$ et je // enfin mon ressenti avec le recul c'était que / surtout pas [rire] // il ne fallait pas aborder ce mot-là ou ce thème-là parce que j'avais vraiment l'impression qu'ils n'étaient pas sûrs de leurs résultats $\downarrow$ [...] on est retournés au service ORL [oto-rhino-laryngologie] [...] $\downarrow$ c'est la première fois que je me suis dit « tiens il y a peutêtre une difficulté $» \downarrow$ autant avant je ne l'avais pas ressentie autant là / je n'étais pas à l'aise du tout $\downarrow$ les infirmières ont réalisé les tests et là // donc on teste chacune des oreilles // et puis elles n'avaient pas l'air sûres d'elles $\downarrow$ [...] le bébé n'était pas dans de bonnes conditions / il n'était pas assez calme / c'était pas fiable / on n'était pas sûrs $\downarrow$ ”

Le témoignage de Marie, loin d'être anecdotique, révèle la part significative de " faux positifs » lors du dépistage, effectué dans les premières heures suivant la naissance, et contre lequel le Comité consultatif national pour l'éthique (CCNE) avait mis en garde lors de la publication en 2006 de l'avis $\mathrm{n}^{\circ} 103$ Éthique et surdité de l'enfant: éléments de réflexion à propos de l'information sur le dépistage systématique néonatal et la prise en charge des enfants sourds. Le nombre important de diagnostics erronés pose la question de la précocité de dépistage. Dans son rapport, le CCNE indique :

Outre le risque de compromettre inopportunément la qualité des premiers contacts de l'enfant avec ses parents, cette stratégie de dépistage en maternité se heurte à une sérieuse difficulté : le risque de faux positifs (suspicion erronée d’un déficit non réel) est bien plus grand dans les premières heures suivant la naissance de l'enfant. [...] Cela laisserait prévoir actuellement de 8000 à 40000 suspicions infondées par an en France, c'est-à-dire 90 à $98 \%$ de diagnostics erronés de déficience auditive parmi les enfants testés alors que 800 à 1000 par an seront réellement sourds. Ce taux de faux positifs s'effondre lors de la répétition des examens dans les premiers jours. (p. 8, note 7)

Face à l'impossibilité de poser un diagnostic immédiat, on peut se poser la question de l'urgence d'un tel dépistage. Dans son témoignage, Marie revient sur les différents rendez-vous lors desquels différents tests sont effectués. Au fur et à mesure des interventions médicales, Marie prend conscience d'une difficulté éventuelle pour son enfant et, en l'absence d'informations précises, elle évoque son malaise grandissant lors du deuxième rendez-vous chez I'ORL.

Marie : " donc là // en fait on avait la sensation que // on a commencé à avoir la sensation qu'ils étaient en train de détecter quelque chose qu'ils ne voulaient pas nous dire $\downarrow$ ou qu'ils voulaient être sûrs avant de nous le dire $\downarrow$ comme on a senti ce doute / nous-mêmes on s'est mis à douter / mais sans que des mots très concrets soient posés dessus $\downarrow$ et ça c'était vraiment avec l'infirmière / le médecin ORL n'était pas du tout à la consultation $\downarrow$ il n'y avait pas tellement d'accompagnement un petit peu psychologique $\downarrow$ voilà on venait d'avoir un bébé / il avait trois semaines / on était en train de faire des tests $\downarrow$ ce n'est pas la chose habituelle quand on a un nouveau-né donc // c'était très inconfortable comme situation $\downarrow$ »

Dans l'attente d'un diagnostic définitif, l'expérience des tests engendre une période d'anxiété pour les parents qui, une fois l'annonce d'un premier test "non concluant", multiplient les rendez-vous validant ou invalidant la première suspicion de défaillance de l'audition. Le CCNE rappelle qu'en cas de suspicion de surdité en période néonatale, "l'examen audiophonologique sera répété dans les 48 heures, au bout de 15 jours, et au plus tard 2 ou 3 mois après la naissance " (p. 8). Cette anxiété est d'autant plus forte que l'une des caractéristiques du dépistage de surdité est qu'il implique une annonce progressive : "les parents vont être confrontés à une succession d'annonces, souvent inscrites elles-mêmes dans le temps (deux à trois mois). Ces différentes annonces vont

\footnotetext{
${ }^{10}$ Tous les noms ont été modifiés.
} 
impliquer différents professionnels. [...] Parfois, les familles peuvent de ce fait avoir l'impression que les professionnels ne disent pas la même chose ou que les informations sont contradictoires, ce qui augmente leur détresse et les confronte à un sentiment d'extrême solitude » (Soriano, 2011, p. 80).

Du côté des professionnels, la responsabilité de l'annonce peut expliquer l'extrême précaution à ne pas nommer la surdité, comme l'explique Marie dans son témoignage, à garder en suspens les orientations du diagnostic quelles qu'elles soient: "Les professionnels ont bien conscience du potentiel traumatique de cette annonce sur les familles, ce qui peut avoir un impact sur leur attitude. Annoncer un diagnostic de surdité si précocement est une fonction à forte composante émotionnelle et peut entraîner des effets de saturation ou des contre-attitudes comme trop d'empathie ou pas assez " (Soriano, 2011, p. 83). Ces attitudes et contre-attitudes véhiculent un stress supplémentaire pour les familles, qui attendent dorénavant une réponse, car l'autre caractéristique de l'annonce du diagnostic de surdité est qu'une réponse va être donnée sans qu'il y ait eu de questionnement initial.

\begin{abstract}
Marie : «Et puis elles ont refait les tests à plusieurs reprises jusqu'à nous dire « bon bah écoutez / sur l'oreille droite il n'y a aucun souci on a un signal / donc c'est déjà ça » $\downarrow$ vraiment texto hein $\downarrow$ "son oreille droite fonctionne / c'est déjà ça $\downarrow$ sur son oreille gauche on a un doute " $\downarrow$ voilà $\downarrow$ et puis on a été lâchés dans la nature comme ça $\downarrow$ [...] le rendez-vous s'est terminé comme ça $\downarrow$ lâchés dans la nature / pas d'explication du médecin ORL // [...] déjà c'était une entrée dans ce monde-là qu'on ne connaissait pas / qui commençait à devenir un petit peu difficile parce qu'on ne savait pas vraiment / on n'était pas sûrs / et avec un médecin qui était complètement // je ne sais pas comment dire / hermétique à tout $\downarrow$ pas du tout psychologue et je trouve que dans le parcours / dans le parcours initial je trouve que vraiment ce qui a manqué / c'était cet accompagnement psychologique $\downarrow$ ne pas balancer comme ça à une famille / voilà votre enfant est peut-être sourd et puis point barre on reprend rendez-vous dans un mois et c'est fini $\downarrow$ je trouve que ce n'est pas possible de laisser des parents / en plus entendants qui n'y connaissent rien / qui ne savent pas du tout pourquoi l'enfant peut être ou ne pas être sourd $\downarrow$ ça ne va pas / vraiment dans le protocole il y a quelque chose qui ne va pas $\downarrow$ »
\end{abstract}

La précocité d'un diagnostic et d'une annonce de surdité devrait en principe, n'ayant pas été amorcées par les questionnements des parents eux-mêmes, impliquer une attention extrême à leur égard : " L'annonce du handicap réclame de l'humanité et de la compétence de la part des professionnels; elle est un moment fort dans la construction des liens entre les parents et leurs enfants » (Zinschitz, 2007, p. 91). Moment d'autant plus fort pour les parents que l'annonce et plus tard l'accompagnement puis l'information vont conditionner fortement les choix que les parents seront amenés à effectuer en termes de modalités linguistiques et pédagogiques de communication pour leur enfant.

Deux approches sont fréquemment développées au sein des discours liés à la surdité, qui rendent compte de l'extrême diversité des situations sociolinguistiques et anthropologiques. L'approche dite " déficitaire " renvoie au manque d'audition de la personne sourde, qui doit être corrigée; l'approche culturelle (ou anthropologique) renvoie quant à elle aux Sourds en tant que communauté linguistique et culturelle (Bédoin, 2018). Ce regard anthropologique introduit la personne Sourde, "sans pudeur, ni euphémisation, comme un être enraciné dans un corps différent " (Gaucher, 2010, p. 17), et rend compte de la manière dont les Sourds s'identifient au monde. La langue des signes, reconnue officiellement en France depuis la loi du 11 février 2005, "pour l'égalité des droits et des chances, la participation et la citoyenneté des personnes handicapées" (Légifrane, 2005) est le vecteur linguistique d'une culture et d'une communauté des Sourds ${ }^{11}$. Pourtant, les parents entendants découvrent généralement la surdité sans avoir jamais été confrontés auparavant aux questions anthropologiques, psychologiques et sociales qu'elle soulève, ni avoir jamais rencontré de personnes sourdes. L'annonce de la surdité aux parents entendants a fait l'objet de différentes études, où les thèmes du "traumatisme", de la "sidération parentale " et de la "détresse psychologique", entraînant une "grave dépression parentale " (Lavigne, 2003), contrastent fortement avec les désirs d'émancipation et de reconnaissance des Sourds.

\footnotetext{
${ }^{11}$ Le $\mathrm{S}$ majuscule fait entrer les Sourds en tant que communauté sociolinguistique à part entière, s'opposant aux personnes sourdes déficientes auditives.
} 
La suite du processus de dépistage est décrite comme violente par Marie, qui évoque « des conditions atroces » et détaille de manière extrêmement précise le souvenir de l'annonce :

Marie : «Barbara était hyper agitée / elle n'arrivait pas à s’endormir dans un sommeil profond / elle se réveillait tout de suite $\downarrow$ en fait l'ORL a commencé à // à devenir assez nerveuse / assez tendue et le / le bilan PEA [Potentiel évoqué auditif] a duré je sais plus quelque chose comme deux heures $\downarrow[\ldots]$ je vous le dis parce que ça nous a choqués // on la voyait réagir par mimiques en fait / assez / désagréables ses mimiques comme si quelque chose n'allait pas $\downarrow$ et nous on était à côté / on n'était pas dans une salle d'attente on était à côté de Barbara et on savait pas ce qui se passait $\downarrow$ et quand on lui posait des questions elle ne nous répondait pas / ou elle était là « je suis pas sûre / il faut qu'on refasse $\downarrow$ sans rien nous expliquer $\downarrow$ « et puis de toute façon c'est pas fiable // » $\downarrow$ donc elle avait fixé une électrode derrière l'oreille avec un sparadrap // et comme Barbara avait des petits cheveux elle nous disait « vous voyez bien avec les cheveux ça tient pas / c'est pas possible de réaliser le test comme il faut » $\downarrow$ enfin bon / des conditions // atroces $\downarrow$ on a commencé franchement // enfin moi j'ai commencé à paniquer à ce moment-là en fait / parce que je me suis dit // je sentais que quelque chose n'allait pas et puis au niveau de / comment dire / du soutien qu'on pouvait avoir par le médecin c'était vraiment / c'était plus que limite $\downarrow$ "

Ces propos, exprimant détresse et colère, que l'on retrouve dans la majorité des témoignages des parents, rappellent l'attention particulière que nécessite l'annonce de la surdité : les mots et les comportements des professionnels ont un impact durable sur les parents et leurs rapports à leurs enfants. En effet, " si les médecins connaissaient l'impact des paroles sur le vécu ultérieur des parents, et par conséquent sur le devenir de l'enfant, ils seraient peut-être plus prudents. Ces mots-là marquent ; ils laissent des traces que le temps n'efface pas. » (Sausse, 1996, p. 25).

Marie : " et puis finalement au bout de deux heures / elle nous a dit comme ça / elle griffonnait sur un bout de papier / « bon ben / il semble qu'il y ait un risque de surdité » $\downarrow$ voilà comme ça $\downarrow$ je vous assure / comme ça $\downarrow$ nous on était là / assis sur un pauvre tabouret à côté de Barbara /// sans aucune psychologie / elle nous a dit ça et hop c'était fini / on va au secrétariat on prend un prochain rendez-vous $\downarrow[\ldots]$ et ça je ne vous l'ai pas dit c'est qu'après on sort de la cabine / donc on va dans son cabinet pour [...] et puis elle commence à me dire // parce qu'à l'époque j'allaitais Barbara et c'est vrai que j'avais beaucoup maigri / j'étais fatiguée / I'allaitement tout ça $\downarrow$ et elle me dit « mais est-ce qu'elle mange bien $\uparrow$ peutêtre qu'elle a un retard de croissance $\uparrow$ il y a peut-être un retard de croissance au niveau de son oreille » $\downarrow$ limite // $\downarrow$ c'était presque ma faute en fait $\downarrow$ »

Au-delà des mots et du sentiment de culpabilisation générés par les questions de l'infirmière, perçues comme déstabilisantes et intrusives par Marie, le lieu de l'annonce est une composante à intégrer dans l'accompagnement afin que ces parents comprennent mieux l'information qui suivra l'annonce du diagnostic. La surdité de Barbara est annoncée à ses parents d'une manière vécue comme expéditive. En l'absence d'un temps d'échanges, ces derniers sont invités à prendre un nouveau rendez-vous. D'autres familles interrogées décrivent la violence ressentie au moment de l'annonce et la nécessité impérative d'un moment d'échange :

Sophie : « Le diagnostic nous a été annoncé de façon // pas psychologue du tout du tout $\downarrow$ vraiment ça a été balancé comme ça $\downarrow$ fin de l'examen on nous a dit / "oui il y a un problème" $\downarrow$ on n'a pas eu // "on va se poser / on va s'asseoir / on va discuter" // non non $\downarrow$ au contraire en fait $\downarrow$ elles ont repris la petite pour faire des examens / la petite pleurait / moi je pleurais $\downarrow$ enfin // $\downarrow$ voilà / ça a été // franchement je trouve qu'en terme de psychologie // sur l'annonce du diagnostic ce n'est pas ça du tout du tout $\downarrow$ ce sera un très mauvais souvenir $\downarrow$ ”

Cette précipitation ressentie par Sophie semble avoir eu pour conséquence d'augmenter son anxiété et son sentiment d'isolement: "Autrefois, le contact entre la mère et l'équipe de la maternité était suffisamment prolongé pour permettre non seulement une explication minimale des tests, mais encore un accompagnement des conséquences psychologiques entrainées par la révélation. Aujourd'hui, les conditions dans lesquelles un enfant vient au monde ne sont plus les mêmes. Ce qui a été gagné en termes de sécurité sanitaire a été perdu en termes de durée et d'échanges » (CCNE, 2006, p. 8).

L'annonce du diagnostic est un moment capital dans le parcours des parents. Ce premier contact avec le " handicap» à travers les professionnels constitue le point nodal qui va conditionner en grande partie les étapes qui vont suivre et le choix que les parents vont faire pour leur enfant, en termes de modèle de communication et plus tard en termes de scolarisation. 


\section{Hiérarchisation de l'information et esseulement des parents : une diversité de solutions escamotée}

À la suite de l'annonce du diagnostic, les parents sont généralement orientés vers une structure médico-sociale ayant pour mission d'accompagner et d'informer les parents sur les choix possibles de communication et de scolarisation pour leurs enfants. II arrive parfois que cette prise en charge ne soit pas immédiate, les familles se retrouvant alors pendant plusieurs semaines, voire plusieurs mois sans conseils ni suivi.

Lucie : ça arrive finalement tard dans le parcours de parent / ça arrive tard par rapport au moment où on apprend la surdité de son enfant et puis le moment où il est pris en charge par une structure / il s'écoule quand même / plusieurs mois $\uparrow$ pour Pierre / c'était au bout d'un an $\downarrow$ il s'est écoulé un an peut-être un petit peu moins et en fait / nous ce qui nous a le plus apporté c'est les infos qu'on est allés chercher par nous-mêmes dans ce laps de temps $\downarrow$

Cette attente fragilise d'autant plus les relations entre les parents et leur enfant qu'elle fait suite à une première période d'attente angoissée, celle du diagnostic, présentée précédemment. Ce deuxième temps de latence intervient en fait alors que des informations ont été déjà transmises par des professionnels à l'hôpital. Au moment de l'annonce de surdité, il arrive fréquemment qu'une première information soit donnée par le médecin ORL, généralement en faveur de l'implantation cochléaire.

Lucie : dès le premier rendez-vous ils te parlent de l'implant $\downarrow$ pour nous ce n'était pas le premier / plutôt le deuxième / mais dès qu'il a été question de // que peut-être il y avait un problème d'audition ou de surdité / on nous a tout de suite parlé effectivement de l'appareillage et très vite on nous a dit "oui mais vous savez il y a aussi l'implant cochléaire qui est possible" [...] ils nous ont expliqué qu'en fonction des types de surdité il y avait des solutions de / qu'ils appellent de réhabilitation auditive / c'est très joli // "de réhabilitation auditive" // je déteste ce terme $\downarrow$ et donc / dans le cas où // entre autres dans le cas d'une surdité profonde il y avait l'implant cochléaire $\downarrow$ mais donc ça a été évoqué tout de suite // très vite $\downarrow$

Plusieurs mois peuvent ensuite s'écouler entre ce temps de l'annonce et de l'information à propos de la réhabilitation auditive et le temps de suivi par les structures médico-sociales qui ont pour mission notamment d'informer sur la diversité des modèles de communication, modèle vocal par une réhabilitation auditive impliquant une implantation cochléaire ou un appareillage, ou modèle de communication en langue des signes. Ce décalage dans l'accès à l'information induit une hiérarchie qui privilégie la réhabilitation auditive.

En 2010, la Haute Autorité de Santé (HSA) a formulé des recommandations de "bonnes pratiques » sur l'accompagnement des familles d'enfants sourds (HSA, 2010). L'ensemble des acteurs interrogés lors de la rédaction de ce document (professionnels de santé, associations de parents d'enfants sourds, associations de personnes sourdes, centres d'information) réclament «l'existence d'un lieu neutre unique où les parents pourraient recevoir l'ensemble des informations relatives à la surdité de leur enfant, quelles que soient les diversités cliniques et éducatives $»$.

Actuellement, en l'absence d'un tel lieu, les familles sont d'abord informées de l'existence de l'appareillage et de l'implant cochléaire à l'hôpital, avant même parfois d'être prises en charge par une structure responsable de l'accompagnement de leur enfant. Il semble que, mis à part la volonté par les parents d'aller à la recherche d'information par eux-mêmes, les premières informations qui leur sont fournies les orientent systématiquement en faveur d'une réhabilitation auditive, soit vers une solution biomédicale. II arrive qu'en cas d'impossibilité de prise en charge immédiate par une structure médico-sociale, certains parents soient envoyés chez un audioprothésiste.

Nathalie : ça ne tombe / pas au bon moment on va dire $\downarrow$ c'était le 30 juin / c'était fin juin début juillet le diagnostic et / là normalement on doit être orienté vers une structure $\downarrow$ la structure ferme l'été $\downarrow$ donc ça c'est la grosse angoisse $\downarrow$ on a été orientés assez vite vers un audioprothésiste / toujours celui qu'on a / qui nous a pris assez rapidement $\downarrow$ mais // mais on se disait « ça fait un an qu'il ne nous a pas entendus / qu'est-ce qu'on va faire / comment il va se saisir de ses appareils » $\uparrow$ il y avait une multitude de questions qui s'ouvraient et il n'y avait personne $\downarrow$

Cette hiérarchisation des possibilités, le médical primant sur l'aspect social et relationnel de la surdité, oriente nécessairement le choix des parents. À l'opposé des discours préconisant une réhabilitation auditive exclusive, 
certains parents pointent également la militance de certaines structures en faveur de la langue des signes. Les recommandations de certains professionnels "pro-langue des signes " sont vécues comme une intrusion et une nouvelle culpabilisation dans leur rôle de parents.

Carine : j'ai eu de la colère avec des professionnels qui des fois ont été // durs ou / qui ne nous ont pas / suffisamment soutenus ou / jugés parfois // comme des parents qui n'acceptaient pas la surdité / qui ne voulaient pas de langue des signes etc. $\downarrow$ »

Ces différents témoignages rejoignent les recommandations de la HAS concernant la nécessité d'un lieu " neutre " d'information, hors de l'hôpital ou des associations militant en faveur de la langue des signes en tant que langue " unique » des enfants sourds. Une information complète et diversifiée à propos des différentes modalités de communication est essentielle afin de donner aux parents la possibilité d'effectuer un choix éclairé, non tributaire des orientations linguistiques de certains organismes et associations. Faire intervenir des médiateurs hors du champ médical lors de cette annonce pourrait permettre de dissocier la surdité d'un aspect exclusivement médical ou culturel. Actuellement, l'information sur l'implant cochléaire est généralement donnée en priorité par les professionnels de santé, au moment de l'annonce. Plus tard des informations complémentaires sont apportées, notamment sur la langue des signes, considérée alors plutôt comme une langue tremplin, c'est-à-dire moins comme une langue à part entière que comme une langue permettant l'accès au français vocal (Millet, 1999; 2004). Pour ces différentes raisons, il nous semble essentiel qu'une information claire et dénuée de militantisme soit proposée le plus tôt possible aux parents, qui se disent parfois submergés d'informations contradictoires, sur Internet, à travers les rencontres avec des membres d'associations, décrits comme étant " pro langue des signes" ou " pro implantation ». Si certains parents se laissent porter par les seuls conseils apportés par les professionnels lors des rendez-vous, la plupart des familles rencontrées lors des entretiens cherchent au contraire à obtenir des informations complémentaires leur permettant d'effectuer un choix conscient entre la diversité des modèles de communication à leur disposition.

\section{Conclusion}

À travers les témoignages des parents, et plus particulièrement à travers le récit de trois mères, Marie, Sophie et Lucie, nous avons tenté de montrer l'importance de l'accompagnement suite à l'annonce de la surdité de leur enfant et surtout la nécessité de concevoir un lieu d'information unique concernant les différentes possibilités de communication. La diversité des situations familiales, la diversité des conceptions de la surdité et celle des solutions proposées pour construire la communication, qu'elles soient cliniques, linguistiques ou éducatives, justifient la création d'un tel lieu, qui permettrait aux parents de se saisir pleinement des enjeux spécifiques à chacun de ces choix. Car ils engagent non seulement la communication mais aussi la scolarisation, et surtout les représentations et pratiques sociales de leurs enfants. C'est la construction de l'identité sociale de leurs enfants qui est en jeu. L'identification précoce de la surdité permet d'aménager l'entrée dans les problématiques qui se posent dans les liens entre enfants sourds et parents entendants. Ces derniers sont amenés à faire un choix par rapport à l'implantation cochléaire, dès les premières semaines après la naissance, et n'ont parfois que très peu de temps pour comprendre et accepter les enjeux de la surdité, ainsi que pour appréhender les diverses modalités de communication orales et signées qui pourraient leur être proposées. Ces choix ne sont pas définitifs mais conditionnent les premières chances d'accès à la communication, et exigent à ce titre, dès l'annonce du diagnostic, une information et un accompagnement les plus éclairés possible. L'analyse des différents entretiens révèle une distension importante et préoccupante entre les préconisations émises par le ministère de l'Éducation nationale, le CCNE et la HAS notamment, et les réalités vécues et décrites par les parents interrogés. Ces contradictions, engendrant des écarts entre recommandations officielles des divers organismes concernés par le traitement éthique de la surdité et les pratiques effectives des équipes, peuvent être interprétées comme un manque de coordination ou de coopération entre les professionnels (médicaux, médico-sociaux et éducatifs) et les familles, que les suggestions que nous avons avancées dans cet article pourraient contribuer à réduire.

\section{Références bibliographiques}

Bardin, L. (2013). L'analyse de contenu. Paris : Presses universitaires de France.

Bédoin, D. (2018). Sociologie du monde des sourds. Paris : La Découverte. 
Bouvet, D. (2003). La parole de l'enfant sourd. Pour une éducation bilingue de l'enfant sourd. Paris : Presses universitaires de France.

Bouvet, D. (2004). Le bilinguisme langue vocale et langue des signes, un luxe ou une nécessité ?. Dans Le bilinguisme aujourd'hui et demain, Actes de la journée d'études et de recherches sur la surdité (p. 71-82). Paris : Éditions du CTNERHI.

Comité Consultatif National d’Ethique pour les Sciences de la Vie et de la Santé (CCNE) (2006). Avis $n^{\circ} 103$ : Éthique et surdité de l'enfant : éléments de réflexion à propos de l'information sur le dépistage systématique néonatal et la prise en charge des enfants sourds [En ligne]. http://www.ccneethique.fr/sites/default/files/publications/avis103.pdf

Dagron, J. (1999). Les Sourds et les Autres. Dans P. Gruson et R. Dulong (dir.), L'expérience du déni (p. 83-91). Paris : Maison des sciences de l'Homme.

Gaucher, C. (2010). Introduction. Dans C. Gaucher et S. Vibert (dir.), Les Sourds : aux origines d'une identité plurielle (p. 17-21). Bruxelles : Peter Lang.

Haute Autorité de Santé (HAS) (2010) Surdité de l'enfant : accompagnement des familles et suivi de l'enfant de 0 à 6 ans [En ligne]. https://www.has-sante.fr/portail/upload/docs/application/pdf/2010-

03/surdite_de_lenfant_-_0_a_6_ans_-_recommandations.pdf

Jones, E. (1989). Evaluating the success of deaf parents. American Annals of the Deaf, 134(5), 312-316.

Kerbrat-Orecchioni, C. (2005). Le discours en interaction. Paris : Armand Colin.

Lavigne, C. (2003). Handicap et parentalité. La surdité, le handicap mental et le pangolin. Paris : Éditions du CTNERHI.

LegiFrance (2005). Loi n²005-102 du 11 février 2005 pour l'égalité des droits et des chances, la participation et la citoyenneté des personnes handicapées [En ligne]. https://www.legifrance.gouv.fr/affichTexte.do?cidTexte=JORFTEXT000000809647\&categorieLien=id

LegiFrance (2014). Arrêté du 3 novembre 2014 relatif au cahier des charges national du programme de dépistage de la surdité permanente néonatale [En ligne]. https://www.legifrance.gouv.fr/affichTexte.do?cidTexte=JORFTEXT000029754753

Meynard, A. (2016). Des mains pour parler, des yeux pour entendre. La voix et les enfants sourds. Paris : Érès.

Millet, A. (1999). Orthographie et écriture, langage et surdité, système, représentations, variations (HDR Sciences du Langage, Université Stendhal, Grenoble).

Millet, A. (2004). La langue des signes français (LSF) : une langue iconique et spatiale méconnue. Cahiers de I'APLIUT, 13(2), 31-44.

Mucchielli, R. (2006). L'analyse de contenu des documents et des communications. Paris : Les éditions ESF.

Rannou, P. (2018). Sociolinguistique de la surdité, didactisation de la pluralité linguistique. Parcours de parents entendants en France et regards croisés sur la scolarisation des élèves sourds: France - États-Unis (Thèse de doctorat, Université Rennes 2/Columbia University).

Soriano, V. (2011). Réflexions sur l'annonce du diagnostic précoce de surdité. Empan, 3(83), 79-85.

Sausse, S. (1996). Le miroir brisé. L'enfant handicapé, sa famille et le psychanalyste. Paris : Calmann-Lévy.

Virole, B. (2006) [1996], Psychologie de la surdité. Paris/Bruxelles : De Boeck Supérieur.

Zinschitz, E. (2007). L'annonce d'un handicap : le début d'une histoire. Approche Centrée sur la Personne. Pratique et recherche, 6(2), 82-93.

Alterstice - Revue Internationale de la Recherche Interculturelle, vol. $7, n^{\circ} 2$ 